\title{
Nanoscale
}

A) Check for updates

Cite this: Nanoscale, 2021, 13, 15422

\section{Multicompartment dendrimicelles with binary, ternary and quaternary core composition $\uparrow$}

\author{
Rebecca Kaup, $t^{a}$ Jan Bart ten Hove, $t^{\mathrm{a}}$,b Anton Bunschoten, ${ }^{a}$ \\ Fijs W. B. van Leeuwen (D) a,b and Aldrik H. Velders (D) *a,b,c
}

Received 13th July 2021 Accepted 27th August 2021 DOI: $10.1039 / \mathrm{d} 1 \mathrm{nr} 04556 \mathrm{c}$ rsc.li/nanoscale

\begin{abstract}
Hierarchically built-up multicompartment nanoaggregate systems are of interest for, e.g., novel materials and medicine. Here we present a versatile strategy to generate and unambiguously characterize complex coacervate-core micelles by exploiting four different dendrimeric subcomponents as core-units. The resulting mesoscale structures have a hydrodynamic diameter of $50 \mathrm{~nm}$ and a core size of $33 \mathrm{~nm}$, and host about thirty 6 th generation polyamidoamine (PAMAM) dendrimers. We have used FRET (efficiency of $\sim 0.2$ ) between fluorescein and rhodamine moieties immobilized on separate PAMAM dendrimers (G6-F and G6-R, respectively) to prove synchronous encapsulation in the micelle core. Tuning the proximity of the FRET pair molecules either by varying the G6-F : G6-R ratio, or by co-assembling non-functionalized dendrimer (G6-E) in the core, reveals the optimal FRET efficiency to occur at a minimum of $70 \%$ loading with G6-F and G6-R. Additional co-encapsulation of 6th generation gold dendrimer-encapsulated nanoparticles (G6-Au) in the micelle core shows a dramatic reduction of the FRET efficiency, which can be restored by chemical etching of the gold nanoparticles from within the micellar core with thiols, leaving the micelle itself intact. This study reveals the controlled co-assembly of up to four different types of subcomponents in one single micellar core and concomitantly shows the wide variety of structures that can be made with a well-defined basic set of subcomponents. It is straightforward to design related strategies, to incorporate inside one micellar core, e.g., even more than 4 different dendrimers, or other classes of (macro)molecules, with different functional groups, other FRET pairs or different encapsulated metal nanoparticles.
\end{abstract}

\section{Introduction}

Block copolymer micelles find wide attention as versatile materials, e.g., for use in medicinal diagnostics and therapeutics, as well as for biomimetic alternatives to compartmentalization and transport strategies found in nature. ${ }^{1-4}$ Strategies for the formation of block copolymer micelles can employ different self-assembly driving forces (e.g., hydrophobic, electrostatic, coordination, hydrogen bonding, etc.); among these, the most applied are hydrophobic and electrostatic interactions. ${ }^{1,2,5-8}$ Micelles based on interaction of oppo-

\footnotetext{
${ }^{a}$ Laboratory of BioNanoTechnology, Wageningen University \& Research, Bornse Weilanden 9, 6708 WG Wageningen, The Netherlands. E-mail: aldrik.velders@wur.nl ${ }^{b}$ Interventional Molecular Imaging Laboratory, Department of Radiology, Leiden University Medical Center, Leiden, The Netherlands

${ }^{c}$ Instituto Regional de Investigacion Cientifica Aplicada (IRICA), Universidad de Castilla-La Mancha, 13071 Ciudad Real, Spain

$\dagger$ Electronic supplementary information (ESI) available: Experimental section; NMR, UV-Vis, fluorescence, DLS, and (cryo-)TEM characterization of micelles with different core compositions; raw data for FRET calculations. See DOI: $10.1039 / \mathrm{d} 1 \mathrm{nr} 04556 \mathrm{c}$

$\$$ These authors contributed equally to the paper.
}

sitely-charged subcomponents have been coined coacervatecore micelles, C3Ms, ${ }^{9,10}$ or poly-ion complex micelles, PICs. ${ }^{11}$ As core-units for the coacervation, linear polymers, branched polymers, proteins, coordination polymers, highly charged coordination complexes, and dendrimers can be efficiently assembled into micelles. ${ }^{4,9,12-19}$

Dendrimers constitute a unique class of polymers that are studied extensively, with poly(AMidoAMine) (PAMAM) dendrimers being among the most explored. ${ }^{20}$ Dendrimers are being extensively investigated for drug delivery purposes, including siRNA delivery. ${ }^{21,22}$ In addition to their well-defined size and 3D-structure, PAMAM dendrimers have interior voids in which small molecules and even metallic nanoparticles can be encapsulated. ${ }^{23-28}$ Dendritic metal complexes are extensively studied for bioimaging. ${ }^{29}$

Multicompartment micelles exhibit several subdomains inside the micelle which can enable and facilitate the solubilization, transport, and subsequent delivery of multiple -potentially (bio)incompatible or toxic- payloads like therapeutic drugs or catalytic payloads. ${ }^{30}$ In theory, the composition of such micelles can be regulated by the statistical mixing ratio of the respective precursors and subcomponents. Different strat- 
egies are being developed to facilitate the design of hierarchical build-up of such structures, ${ }^{1,2,5,6,8,31,32}$ but at the same time, the need for unambiguous characterization of these complex systems becomes of paramount importance. In particular, the quantitative analysis of subcomponents in mesoscale structures is intrinsically challenging.

A few years ago, we developed a strategy to control the number of dendrimers per micelle by combining different generations of negatively-charged or positively-charged dendrimers with oppositely-charged block copolymers under charge-stoichiometric conditions. ${ }^{33}$ Furthermore, we exploited gold nanoparticles embedded inside the dendrimers to investigate micelle characteristics at a single micelle level. $^{34-37}$ This work allowed us to demonstrate that even nanoparticle-containing dendrimers can be assembled into $\sim 50 \mathrm{~nm}$-sized well-defined and stable micelles. ${ }^{37}$ From cryoTEM micrographs, micelle aggregation numbers can be determined by simply counting the number of nanoparticles per micelle, so revealing the by themselves invisible dendrimers. By mixing empty dendrimers and gold-loaded DENdrimers prior to block copolymer addition, the formation of micelles with both types of dendrimers in the core was proven, i.e., binary core composition. As dendrimers are in close proximity of each other inside the densely packed dendrimicelle core ${ }^{34}$ we hypothesized that Förster Resonance Energy Transfer (FRET) could be used to prove and investigate synchronous co-assembly of different building blocks. ${ }^{38-40}$ In fact, we have exploited Förster Resonance Energy Transfer (FRET), in addition to fluorescence spectroscopy, dynamic light scattering (DLS) and cryoTEM, to unambiguously prove the (co)-assembly of up to four different building blocks in a single micelle core.

As shown in Scheme 1 our approach is as follows: we created a set of 6th generation PAMAM dendrimers: regular, i.e., non-functionalized and empty dendrimers (G6-E), fluorescein- (G6-F) and rhodamine B-functionalized (G6-R) dendrimers, and gold-nanoparticle loaded dendrimers (G6-Au). Charge-stoichiometric addition of a poly(methacrylic acid)block-poly(ethylene oxide) block copolymer, $\mathrm{pMAA}_{64}-b$ $\mathrm{PEO}_{885}$, yields dendrimicelles. With the dendrimer building blocks in hand, various mixing strategies allow us to obtain micelles with different core compositions, containing only one type of dendrimer, two different dendrimers, three different dendrimers, or four different dendrimers, coined regular, binary, ternary and quaternary-core dendrimicelles, respectively.

The data obtained from systematic and rational mixing of the various dendrimer building blocks as shown in Scheme 1, unambiguously prove the synchronous co-assembly of, up to four, different dendrimer types into one singular micellar core. The results strongly support and justify the design of complex hierarchically built-up nanoaggregates for, e.g., multicompartment materials and medicinal applications, for which the incorporation of different subcomponents is of paramount importance, e.g., in multimodal imaging, combined drug delivery, or theragnostic applications.

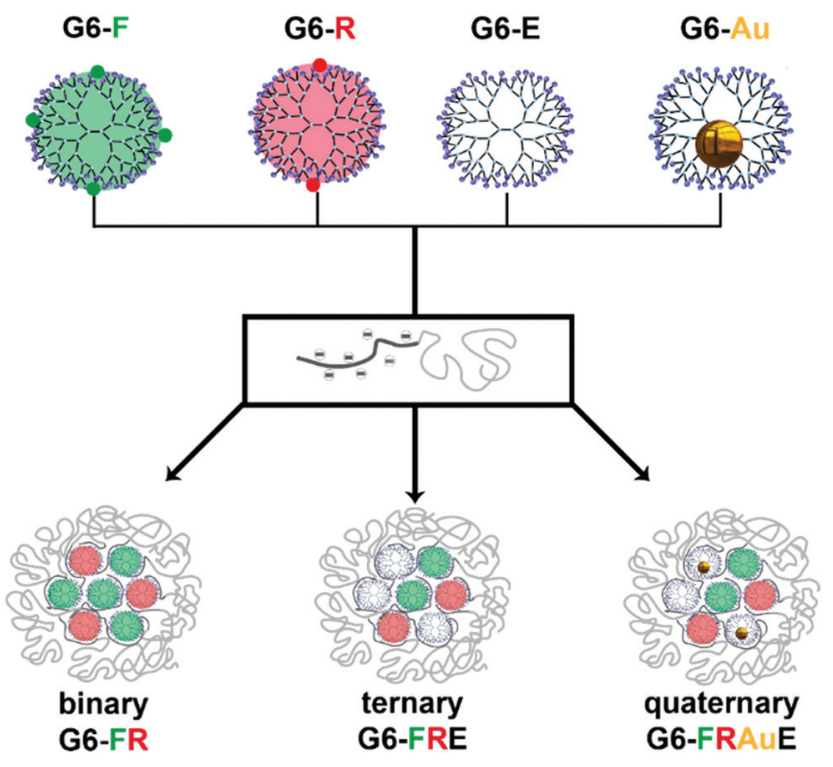

Scheme 1 Schematic overview showing the conversion of regular 6th generation PAMAM (G6-E) into fluorescent fluorescein-functionalized (G6-F), rhodamine B-functionalized dendrimers (G6-R), and dendrimers with encapsulated gold nanoparticles (G6-Au). Charge-stoichiometric addition of negatively-charged $\mathrm{pMAA}_{64}-b-\mathrm{PEO}_{885}$ block copolymer to the positively charged G6 dendrimers results in the formation of complex coacervate core dendrimicelles. The dendrimicelle core composition can be stoichiometrically tuned by changing the mixing ratio of the four different dendrimers prior to block copolymer addition, resulting in binary-core, ternary-core and quaternary-core micelles. The positive charges present on the dendrimer periphery at $\mathrm{pH} 7$ and the charges inside the micelle cores are omitted for clarity reasons. It is important to note that the cartoon of the binary/ternary/quaternarycore structures represents the respective dendrimicelle dimensions and its subcomponents approximately on the same relative scale. The cross section of the dendrimicelles shows 7 dendrimers in close proximity, which in a spherical dendrimicelle core containing about 30 dendrimers is in fact the expected ball-park number; also the overall sizes of the dendrimicelle (50 nanometer diameter: 10 nanometer shell and 30 nanometer core) are on relative scale with the dendrimers; useful to comment that the dendrimers perhaps are slightly more spaced and of course in solution are dynamic.

\section{Results and discussion}

The dendrimicelle strategy described in Scheme 1 allows for a detailed investigation of the formation and composition of coacervate-core micelles, revealing proximity and stability of its building blocks. The different micellar subcomponents were synthesized and characterized following standard literature procedures (see ESI $\dagger$ ). The primary amines located at the periphery of PAMAM dendrimers allow for straightforward conjugation which enables the introduction of functional groups such as fluorophores. ${ }^{41-46}$ Micelle formation is rather robust once the polymer ratios are close to charge stoichiometries, ${ }^{34-37}$ so minor alterations in dendrimer's surface composition are not expected to affect micelle formation. We chose to functionalize PAMAM generation six with only 2-4 fluorophores per dendrimer which corresponds to a 
dendrimer functionalization degree of about $2 \%$ with respect to the number of terminal amines on the dendrimers (i.e., theoretically total of $256-\mathrm{NH}_{2}$ for PAMAM generation 6). We chose this low functionalization degree to affect the chargedriven micelle formation the minimum possible, and to prevent intra-dendrimer self-quenching of fluorophores. ${ }^{47}$ Fluorophore-functionalized dendrimers were obtained by reacting amine-terminated PAMAM dendrimers with fluorescein isothiocyanate (FITC) or rhodamine B-isothiocyanate (RITC) (see Fig. S1† for molecular structures). After purification by dialysis, the dendrimers were characterized with absorbance and steady-state fluorescence spectroscopy and ${ }^{1} \mathrm{H}-\mathrm{NMR}$ (Fig. S2-S4 $\dagger$ ). The fluorescence quantum yield of G6-F dendrimers was about 0.2 , while the quantum yield of G6-R dendrimers was about 0.06 . Table $\mathrm{S} 1 \dagger$ shows an overview of the quantum yield and number of fluorophores per dendrimer, which is 4 and 2 for FITC and RITC, respectively. Dendrimer-encapsulated gold nanoparticles (AuDENs) were synthesized by complexation and reduction of 128 molar equivalents of $\mathrm{Au}^{3+}$ per dendrimer, forming G6-Au (AuDENs of $1.7 \pm 0.4 \mathrm{~nm}$, see Fig. $\mathrm{S} 5 \dagger){ }^{24,34-37}$

With the four different building blocks (G6-F, G6-R, G6-E and $\mathrm{G6} 6 \mathrm{Au}$ ), we systematically investigated the micelle core composition. For all prepared micelles, we have proven the coassembly of the different dendrimers in a single dendrimicelle core assessing core composition (e.g., different ratios of the various types of dendrimers) by fluorescence spectroscopy and FRET. Details about the FRET calculations and all corresponding absorption, excitation and emission spectra and reference experiments are provided in the ESI. $\uparrow$ Below we discuss the data following four steps: (I) describing binary-core compositions with fluorophore-labelled dendrimers and non-functionalized dendrimers, (II) discuss mixing of fluorophore-functionalized and non-functionalized dendrimers in ternary-core micelles, (III) tuning the FRET quenching by co-assembly of AuDENs in quaternary-core systems, and (IV) illustrating the controlled chemical responsive nature of complex multicompartment nanoaggregates.

\section{Defining a binary FRET core micelle with fluorophore- functionalized dendrimers}

In order to exclude possible self-quenching phenomena between fluorophores on neighboring dendrimers, we first studied micelles with a binary core composition, in which either fluorescein- or rhodamine-labelled dendrimers were coassembled with non-functionalized dendrimers (G6-F + G6-E or G6-R + G6-E), and validated the effect of core composition on the fluorescence. We consecutively studied the co-assembly of both, G6-F and G6-R, at different ratios, demonstrating a clear and tunable FRET efficiency.

Fluorescent dendrimicelles with a binary core composition of G6-E and G6-F or G6-R. Micelles were prepared by chargestoichiometric addition of a poly(methacrylic acid)-block-poly (ethylene oxide) block copolymer $\left(\mathrm{pMAA}_{64}-b-\mathrm{PEO}_{885}\right)$ to a dendrimer solution with G6-E and either G6-F or G6-R in various ratios; see Scheme (1) and Fig. 1a. The positively-charged dendrimers and negatively-charged poly(methacrylic acid) block form the complex coacervate core of the dendrimicelle, while the poly(ethylene oxide) blocks prevent macroscopic phase separation. ${ }^{9,15}$ Dynamic light scattering (DLS) confirmed the

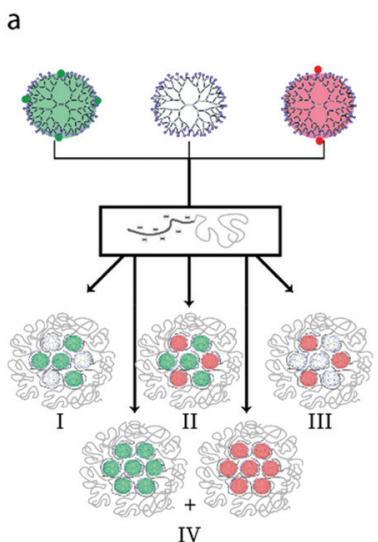

IV b
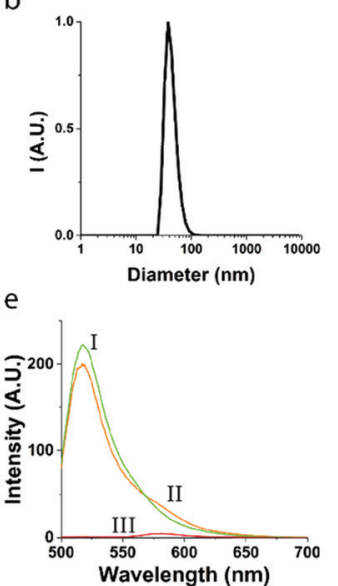

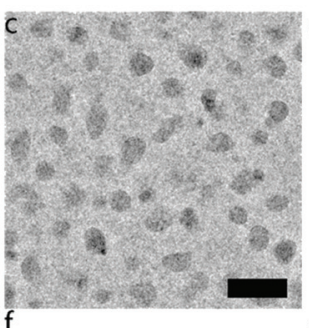

d
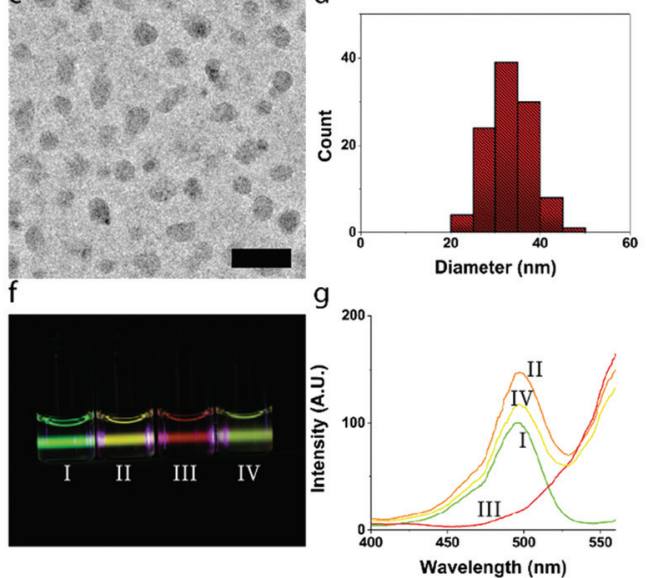

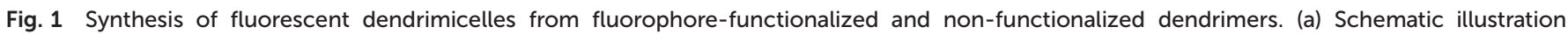
showing the formation of various dendrimicelles from G6-F, G6-R, and G6-E dendrimers, i.e. a 1:1 mix of G6-F: G6-E (sample I), G6-F: G6-R (sample II), G6-R: G6-E (sample III) and G6-F + G6-R (sample IV). (b) The number-averaged Dynamic Light Scattering size plot of dendrimicelle sample II indicates the hydrodynamic diameter of the dendrimicelles to be $\sim 50 \mathrm{~nm}$. (c) CryoTEM micrograph of dendrimicelle sample II. Scale bar represents $100 \mathrm{~nm}$. (d) Size histogram showing the determined dendrimicelle core size distribution, as determined from the cryoTEM micrograph in (c). The average dendrimicelle core size is $33 \pm 5 \mathrm{~nm}$. (e) Fluorescence emission plot showing the emission of the dendrimicelle samples shown in (a): I (green), II (orange) and III (red). The excitation wavelength was $480 \mathrm{~nm}$. (f) Photo visualizing the tunable emission of the micelle samples I-IV shown in (a); excitation was done with a UV laser at $405 \pm 10 \mathrm{~nm}$. (g) Excitation plot of the dendrimicelle samples shown in (a): I (green), II (orange), III (red) and IV (yellow); the emission wavelength was fixed at $580 \mathrm{~nm}$. 
formation of $\sim 50 \mathrm{~nm}$-sized micelles containing G6-F and G6-R in a $1: 1$ ratio (Fig. $1 \mathrm{~b}$ and $56 \dagger$ ). The critical micelle concentration (CMC) of these micelles was $\sim 1 \mathrm{mg} \mathrm{L}^{-1}$ of total polymer concentration, comparable to previously obtained values for generation 6 based dendrimicelles ${ }^{34-37}$ (Fig. S7†). Micelle formation was further confirmed by cryoTEM (Fig. 1c and $d$ and enlarged in Fig. S8 and S9†), revealing the dendrimicelle core size to be $33 \pm 5 \mathrm{~nm}$ and presenting a spherical shape. Both core size and the shape of these dendrimicelles are similar to the dendrimicelles made from non-functionalized or nanoparticle-containing sixth generation dendrimers as reported before. ${ }^{36}$ These results underline that micelle formation is not affected by the (low degree of) functionalization of the dendrimers with the fluorophores. Next, we found that the fluorescence emission of dendrimicelles, containing G6-E and G6-F or G6-R, scales linearly with the functionalized dendrimer fraction in the core (see Fig. S11 $\dagger$ ), corroborating an absence of inter-dendrimer fluorescence quenching after micellization. This holds for both the fluorescein- and rhodamine-functionalized dendrimers in dendrimicelles. Also, micellization itself does negligibly affects the spectral properties of the fluorophore-functionalized dendrimers (Fig. S4 and $\mathrm{S} 10 \dagger)$.

FRET quantification in binary-core micelles composed of coassembled G6-F and G6-R. The fluorophore combination fluorescein/rhodamine-B is a well-known FRET pair, with a Försterradius of about $5.5 \mathrm{~nm}$. As the dendrimers inside the complex coacervate core of the dendrimicelles are densely packed, ${ }^{34}$ coassembly of the fluorophore-functionalized dendrimers inside a single micelle was hypothesized to bring both fluorophores close enough to result in FRET. Fig. 1e shows the steady-state fluorescence spectra of G6-FR micelles (1:1 G6-F:G6-R; II) and the two control samples G6-FE (1:1 G6-F : G6-E; I) and G6RE (1 : 1 G6-R : G6-E; III). The spectra show characteristic emission bands around 520 and $580 \mathrm{~nm}$ for fluorescein and rhodamine, respectively. When comparing the fluorescence intensities of the G6-FR sample with the controls, a decrease of the donor signal and an increase of the acceptor signal is observed, leading to an increase in the acceptor : donor signal ratio, which is characteristic for FRET. Importantly, this increase is not observed when G6-F and G6-R are present in separate micelles, i.e. not combined in one micellar core (Fig. S12 $\dagger$ ). Using formulas (1)-(4) (see Experimental section in ESI $\dagger$ ), we calculated the efficiency of FRET for the G6-FR sample to be $\sim 0.2$. With a Förster radius of $5.5 \mathrm{~nm}$ (ref. 48) this indicates an average donor-acceptor spacing of $\sim 7 \mathrm{~nm}$, about the size of a single generation six dendrimer. The FRET efficiency in the control sample with micelles containing pure G6-F and G6-R cores, and for G6-F and G6-R dendrimers suspended freely in solution (i.e., not embedded in dendrimicelles) was found to be $<0.01$ (see Fig. S13†).

The FRET efficiency was additionally characterized analyzing excitation spectra (Fig. 1g), with the emission wavelength fixed on the acceptor molecule (viz., rhodamine, $580 \mathrm{~nm}$ ). The control sample IV, containing two different dendrimicelles, one with G6-F in the core and one with G6-R, shows the expected spectral sum of the reference dendrimicelles samples I and III. The FRET sample II, on the other hand, with both G6-F and G6-R in the core of a single dendrimicelle, shows the excitation band at $490 \mathrm{~nm}$ much higher than in sample IV or the sum of samples I and III. In other words, once the two fluorophores find themselves in close proximity within the same dendrimicelle core, part of the fluorescein excitation ends up in rhodamine fluorescence. Based on the intensity difference between the FRET sample II and the control sample IV, a FRET efficiency of about $20 \%$ is determined.

For the micelles presented here, it is to note that the block copolymer, the dendrimers, ${ }^{49,50}$ and the functionalization degree of the dendrimers have a statistical (size) distribution. Consequently, the formed dendrimicelles also have a statistical distribution of subcomponents; we showed before that the number of dendrimers per dendrimicelle has a standard deviation of $10 .{ }^{36}$ Therefore, the FRET efficiency reflects the average donor:acceptor distribution and spacing inside the single micelle core, vide infra. Based on the dendrimicelle aggregation numbers determined before, ${ }^{36}$ an average of 30 dendrimers are present in sixth-generation dendrimicelles. At a G6-F fraction of 0.5 , this corresponds to $\sim 15$ G6-F and $\sim 15$ G6-R dendrimers per micelle, corresponding to $\sim 60$ fluorescein- and $\sim 30$ rhodamine-fluorophores per micelle. As FRET efficiency is inversely proportional with the sixth-power of donor-acceptor spacing, this suggests that in practice FRET, with an average spacing of $7 \mathrm{~nm}$, will mainly occur between fluorophores on directly-neighboring dendrimers. Importantly, the presence of FRET in sample G6-FR (II) proves co-assembly of G6-F and G6-R dendrimers inside the formed dendrimicelles. Equally important, the absence of FRET for at least three days, in sample G6-F + G6-R (IV) shows that the corecomposition of these dendrimicelles is kinetically inert, i.e. there is no exchange of dendrimers from one micellar core to another.

Fig. If shows a photograph of the four samples mentioned above (I, II, III and IV) under blue laser light ( $\lambda=405 \mathrm{~nm})$ illumination. The photo illustrates that the micelle emission color can be roughly tuned between green (G6-FE; I), yellow (G6-FR; II) and red (G6-RE; III) by altering the relative amounts of the various fluorophore-labelled dendrimers prior to the addition of the block copolymer and consecutive micelle formation. In addition, there is a fine color difference between G6-FR (II) and G6-F + G6-R (IV) because of the FRET effect between the fluorescein and rhodamine when residing in the same micelle core resulting in a yellowish green fluorescence (G6-FR; II), in contrast to the non-FRET sample (G6-F + G6-R; IV) which has the two fluorophore-labelled dendrimers residing in separate micelles.

\section{Quantitative analyses of FRET aspects in binary and ternary- core dendrimicelles}

Having defined above the FRET system and efficiency of coassembled fluorophore-labelled dendrimers in a micelle core, we next determined the FRET efficiency at varying ratios of the FRET pair subcomponents in binary-core systems. 
Consecutively, we quantified the effect of fluorophore-functionalized dendrimer spacing exploiting ternary-core dendrimicelles that contain a fixed ratio of fluorophore-labelled dendrimers in the core, 'diluted' with additional non-functionalized dendrimers, G6-E.

FRET quantification in binary-core micelles with varying ratios of G6-F and G6-R. A series of dendrimicelles was prepared by mixing G6-F and G6-R dendrimers in various ratios in the core, fraction $x$, respectively $1-x$, while keeping the total dendrimer amount constant (Fig. S14a †). At low G6-F fractions and up to a G6-F fraction $(x)$ of about 0.5 , the determined FRET efficiency inside the dendrimicelles is steady at $\sim 0.2$ (Fig. S14b, $\uparrow$ indicated with a red circle). In this range, a G6-F dendrimer inside the dendrimicelle core is surrounded mainly by G6-R dendrimers, ensuring maximum-obtainable FRET. The maximum FRET is about $20 \%$ and not $100 \%$ in this system due to the spacing of dendrimers inside micelles, the low functionalization degree and orientation of the fluorophores. At G6-F fractions higher than 0.5, the FRET efficiency decreases (see also Fig. S15-S18 $\dagger$ ), which is as expected because of the-on average-diminished proximity of all fluorescein molecules to an acceptor molecule.

Ternary-core micelles composed of co-assembled G6-F, G6-R and G6-E dendrimers. With the efficiency of FRET in binarycore micelles composed of G6-F and G6-R to be constant up to a G6-F fraction of $\sim 0.5$, vide supra, using a G6-F : G6-R ratio of 1 we studied the effect of co-assembly with non-functionalized G6-E dendrimers inside the dendrimicelle core. As FRET is strongly inversely dependent on distance, increasing the average donor:acceptor spacing through co-assembly, i.e. 'diluting', with G6-E was expected to have a FRET-attenuating effect. We co-assembled G6-F, G6-R and G6-E, keeping the ratio G6-F : G6-R constant, and varied the ratio between fluorophore-functionalized (in total: $2 x$ ) and non-functionalized dendrimers $(1-2 x)$ (Fig. 2a). Fig. 2b shows that up to a non-functio- a

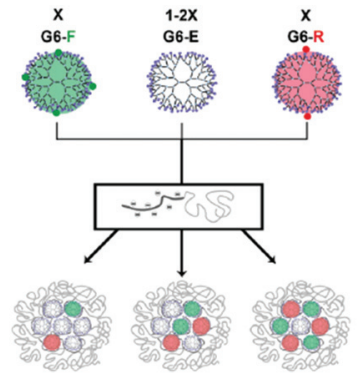

b

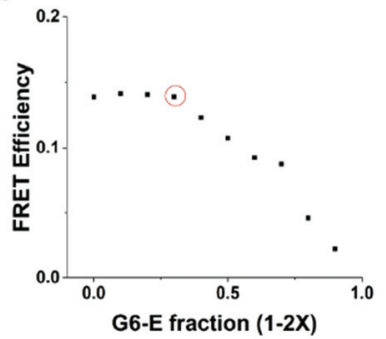

Fig. 2 Effect of the G6-E fraction on the FRET efficiency of the corresponding ternary dendrimicelles. (a) Scheme illustrating the synthesis of ternary-core dendrimicelles with a varying density of fluorophores inside the dendrimicelle core. The fluorophore density in the micelle core is tuned by mixing in non-functionalized dendrimers for the micelle formation, while keeping the ratio of G6-F:G6-R at 1, and keeping the total amount of dendrimers constant. (d) FRET efficiency plot of dendrimicelles made with a varying fraction of non-functionalyzed dendrimers. From this graph, it appears that at non-functionalized dendrimer fraction above 0.3 (indicated with a red circle) the observed FRET efficiency rapidly decreases. nalized dendrimer fraction of $\sim 0.3$ (indicated with a red circle), the FRET efficiency remains constant at $\sim 0.15$, after which it decreases (see also Fig. S19†). In other words, whilst keeping the G6-F/G6-R ratio constant at 1, mixing in G6-E up to $30 \%$ does not affect the FRET efficiency; above $30 \%$ of non-functionalized dendrimer, the average distance between donor-acceptor molecules inside the micelle core increases such that FRET characteristically decreases. Assuming the $\sim 30$ dendrimers in a sixth generation dendrimicelle to be spherical and densely packed, the number of neighboring dendrimers in proximity of a single dendrimer can vary from 12 for a dendrimer in the center of the core, to 2 or 3 for a dendrimer at the periphery of the core. Clearly, at higher fractions of non-functionalized dendrimers, the chance of finding a non-FRET partner as neighbor increases. The attenuated FRET efficiency at G6-E fractions $>0.3$ are likely a result of both the nearest-neighbor composition inside the dendrimicelle core and on the respective orientation of the fluorophores that is of influence. ${ }^{51}$

The results presented in this section show that the efficiency of FRET can be tuned not only by the ratio of the individual fluorophores (i.e., the composition of the neighboring dendrimers inside the micelle core), but also by tuning the average fluorophore-labelled dendrimer spacing, i.e. the dilution with non-labeled dendrimers. Moreover, the nonlinear dependence of FRET on the fluorophore-functionalized dendrimer fraction (Fig. 2) unambiguously proves the ternary co-assembly of G6-F, G6-R, and G6-E dendrimers. The determined optimal FRET pair ratio of $1: 1$ and maximum percentage of additional, i.e., non-functionalized, dendrimers of $30 \%$ not affecting FRET were used as boundary conditions in the consecutive studies with gold-nanoparticles-filled dendrimers.

Dendrimicelles with gold-nanoparticle-containing dendrimers, from binary and ternary to quaternary-core compositions

Gold nanoparticles are known to be able to affect a fluorophore's brightness as result of plasmonic coupling. ${ }^{52,53} \mathrm{We}$ therefore extended the hierarchical complexity of multicompartment micelles by incorporating dendrimer-encapsulated gold nanoparticles, G6-Au, in the formation of dendrimicelles. Indeed we were able to efficiently quench the FRET effects using AuDENs and quantified and tuned the quenching efficiency working with the optimized fluorophore-labeled dendrimer ratio obtained in the previous section.

Co-assembly of Au-nanoparticle filled dendrimers with fluorophore-labelled dendrimers inside micellar cores. To verify the self-assembly behavior of fluorophore-functionalized dendrimers and AuDENs, we prepared a dendrimicelle sample in which we first mixed G6-F and G6-Au dendrimers in a 1:1 ratio, followed by dendrimicelle formation. A representative cryoTEM micrograph of these binary-core dendrimicelles, shows that these micelles contain $15 \pm 5$ nanoparticles per micelle (Fig. S9†). A number that represents 1:1 ratio in the core. $^{36}$ Plasmonic effects of nanoparticles on fluorophores depend on size and shape of the nanoparticle, particle-fluorophore spacing, and spectral overlap between the fluorophore emission and the plasmon. With generation six dendrimers 
being $\sim 7 \mathrm{~nm}$ in diameter, and plasmonic effects proceeding up to about five times the nanoparticle radius, ${ }^{52}$ the $\sim 2 \mathrm{~nm}$ sized dendrimer-encapsulated gold nanoparticles (one AuNP/ dendrimer $)^{24}$ are expected to selectively affect the fluorescence of fluorophores in close proximity. The co-assembly of gold nanoparticles can hence be used to prove the formation of micelles with a quaternary core composition. Hereto, first the plasmonic effect on luminosity and FRET efficiency in ternary core micelles was studied; consecutively, quaternary-core dendrimicelles were prepared containing G6-F, G6-R, G6-Au and G6-E dendrimers inside a single micelle core.

Plasmonic effects of G6-Au in ternary-core (FRET-reduction) co-assembled dendrimicelles. As upfront control experiments, we first prepared dendrimicelles from G6-F and G6-R at a $1: 1$ ratio, co-assembled with either G6-E or G6-Au dendrimers in the remaining fraction of 0.3 (see Fig. S20, $\dagger$ samples I \& II). Compared to the micelles made with G6-E dendrimers, those with G6-Au show a decreased FRET effect. Additionally, we verified whether these interactions extent beyond the micelle core, although based on the dimensions of the dendrimicelle shell $(\sim 7 \mathrm{~nm})^{34}$ no significant plasmon effect is expected. Fig. S20b $\dagger$ compares the emission of ternary-core micelles containing G6$\mathrm{F}$, G6-R, and G6-Au in the same dendrimicelle core to that of binary-core micelles (without gold), mixed with separate G6-Au dendrimicelles. FRET was only seen in the binary-core micelle, showing the effect of the gold nanoparticles on the fluorophore emission not to extend beyond the micellar core, i.e. there is no inter-micellar effect (see also Fig. S20c $\dagger$ ). In short, co-assembly of G6-F and G6-R with G6-Au, rather than G6-E, yields dendrimicelles with a diminished FRET efficiency, in line with expectations. ${ }^{52,53}$

Tuning of FRET in ternary and quaternary dendrimicelles with varying ratio G6-E : G6-Au dendrimers. Above, we established that in a G6-FR-filled micelle-core, co-assembly of G6-E dendrimers up to a fraction of 0.3 does not affect FRET efficiency, whilst co-assembly of G6-Au does. Consequently, the observed FRET efficiencies can be expected to quantitatively reflect the nanoparticle-containing dendrimer fraction. We thus prepared micelles that contain identical amounts of G6-F and G6-R dendrimers ( $f=0.35$ each), and varied the ratio of G6-E: G6-Au dendrimers in the remaining $f=0.3$. Fig. 3a schematically depicts the formed micelles, with Fig. $3 \mathrm{~b}$ displaying the observed FRET efficiencies for the dendrimicelles (see also Fig. S21†). At a G6-Au fraction of 0, (i.e, corresponding to a G6-E fraction of 0.3), the efficiency of FRET is about 0.17 , similar to what observed in Fig. 2 b. When preparing samples in which G6-E is gradually replaced by G6-Au, the FRET efficiency concomitantly decreases, confirming that all four dendrimer types can co-assemble in a single dendrimicelle core. Fig. 3c shows a cryoTEM picture of quaternary-core dendrimicelles containing a G6-Au fraction of 0.15 . The average number of AuNPs per micelle is $4 \pm 2$ in perfect agreement with the expected values for dendrimicelles made from a mix of $70 \%$ fluorescent dendrimers, $15 \%$ empty dendrimers and $15 \%$ nanoparticle-containing dendrimers.

The non-linear behavior of the observed decrease of FRET upon increasing the G6-Au fraction in the dendrimicelles is corroborating the fact that 4 different types of dendrimers are encapsulated synchronously inside a single micelle core. If, hypothetically, a mixing would occur with a preference for the ternary-core micelles, i.e. G6-R/G6-F/G6-E and G6-R/G6-F/G6$\mathrm{Au}$, a linear decrease would have been expected going from a G6-Au fraction of 0 to 0.3 .

\section{Chemical responsiveness and tuning of the ternary-quaternary core composition}

The design of responsive micellar structures constitutes an important area of research because of potential applications
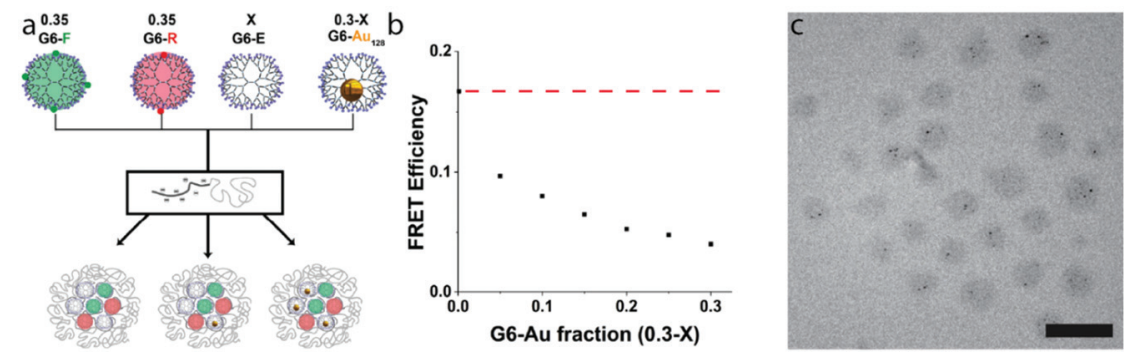

Fig. 3 Co-assembly of three (G6-Au fraction being 0 or 0.3 ) or four (the intermediate fractions) types of dendrimers inside a single dendrimicelle, forming micelles with ternary, respectively, quaternary dendrimer core composition. (a) Scheme showing the synthesis of dendrimicelles containing up to four different dendrimers. The fraction of G6-F and G6-R dendrimers was kept constant at 0.35 each, while the remaining fraction dendrimers ( $f=0.3$ ) was composed of a varying amount of G6-E : G6-Au dendrimers. Note, out of the three dendrimicelle cartoons, the outer ones concern ternary-core compositions with, respectively, only additional empty (left) or Au-NP-filled (right) dendrimers. (b) FRET efficiency graph showing the determined FRET efficiency versus the fraction G6-Au inside a dendrimicelle core (black squares). To aid the eye, the horizontal red dashed line indicates the constant FRET efficiency in case only G6-E would be present rather than G6-Au fractions (i.e. a repetition of the first data point (G6-Au fraction 0)). This graph shows that with increasing amount of nanoparticle-containing dendrimers in the dendrimicelle core, the observed FRET efficiency decreases, corroborating the co-assembly of all four dendrimers inside a single micelle. (c) Representative cryoTEM micrograph of a dendrimicelle sample made from a G6-Au fraction of 0.15 showing that the DENs are evenly divided between the micelles. The average number of AuNPs per micelle is $4 \pm 2$ in perfect agreement with the expected values for dendrimicelles made with $15 \%$ nanoparticle-containing dendrimers. The scale bar represents $100 \mathrm{~nm}$. 
such as catalysis or small-molecule/drug delivery. ${ }^{18,54}$ The PEG shell of the block copolymer provides a relatively flexible barrier that allows the transport in-and-out of the core of small molecules. As there are several chemical ways to decompose gold nanoparticles, this allows the design of responsive multicompartment nanoaggregate systems. Here we investigated the possibility to exploit the quenching effect of the gold nanoparticles on the luminescence of the dendrimicelles. So the micelles themselves remain intact, but the content of the core is altered.

\section{Thiol-etching of G6-Au corroborates quaternary core composition}

Above we showed that the attenuation of the FRET efficiency in quaternary core dendrimicelles scales with the fraction of micelle-embedded G6-Au. Using thiol-mediated etching we intended to degrade the micelle-embedded gold nanoparticles (Fig. 4a) and monitor the impact of etching on FRET efficiency, proving the multicompartment concept of the dendrimicellar core. ${ }^{37,55}$ For this, we prepared dendrimicelle samples containing a total fraction of fluorophore-functionalized dendrimers of 0.7 (i.e., containing 0.35 G6-F and 0.35 G6$\mathrm{R})$, with the remaining dendrimer fraction consisting of a mix of G6-E (respectively, 0.3, 0.15, and 0) and G6-Au (respectively,
$0,0.15,0.3)$. DLS measurements and CryoTEM on a thioletched sample confirm that $\sim 50 \mathrm{~nm}$-sized dendrimicelles are still present in solution after etching (Fig. $4 \mathrm{~b}$ and c and $\mathrm{S} 22 \dagger$ ). We determined the FRET efficiency in the respective micelles, before and after incubation with excess of mercaptoethanol to gold. Fig. 4d shows (in black) the FRET efficiency before thioletching of G6-Au. After thiol-etching the FRET efficiency (in orange), in the samples that contained G6-Au, increased from $<0.05$ to $\sim 0.12$. Fig. 4 e shows an increase in fluorescence emission around $580 \mathrm{~nm}$ for the sample made with a G6-Au fraction of 0.3 after thiol etching (in orange) compared to before (in black) (see also S23†). This emission recovery corresponds to degradation of the micelle-embedded gold nanoparticles, with concomitant loss of the plasmonic attenuation of the FRET efficiency. The observed FRET efficiency of the control sample made with a G6-E fraction of 0.3 (i.e., no G6-Au present) decreased slightly, from 0.17 to 0.14 , implying that mercaptoethanol has a moderately deteriorating effect on G6-F and/or G6-R luminosity, explaining why the FRET efficiency is not fully recovered in the sample with G6-Au fractions of 0.15 and 0.3. Fig. 4f displays a photograph of dendrimicelle samples under UV-illumination $(\lambda=365 \mathrm{~nm})$. The cuvettes contain dendrimicelles made with a G6-Au fraction of 0.3
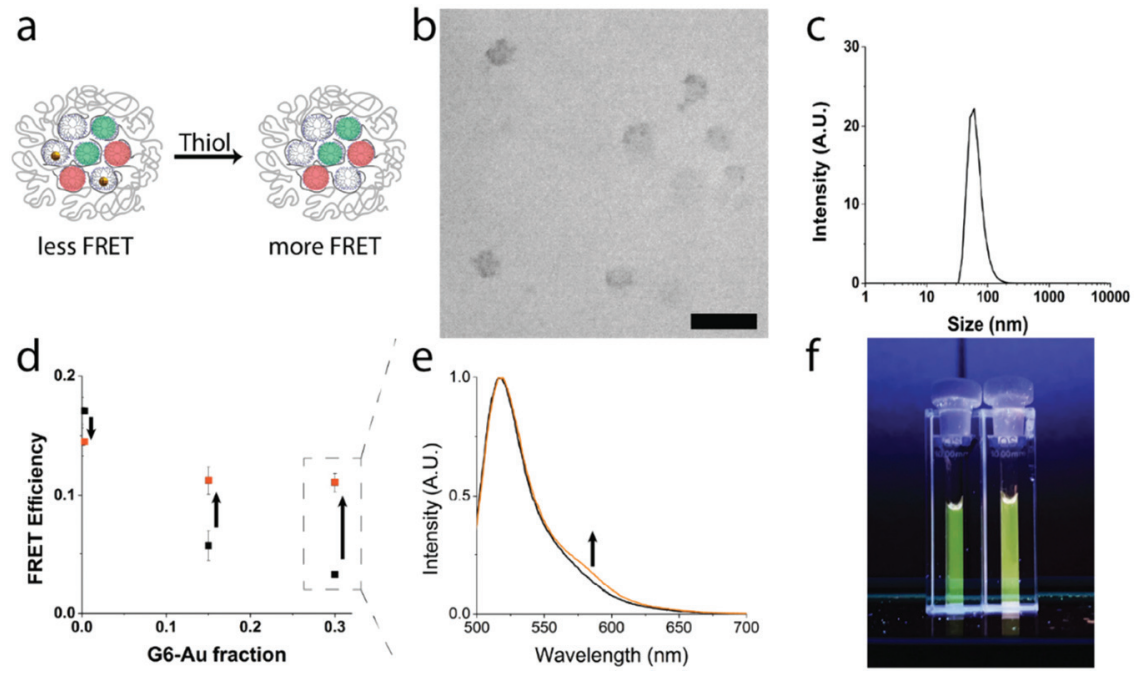

Fig. 4 FRET recovery upon thiol-induced etching of micelle-embedded gold nanoparticles. (a) Schematic illustration showing the etching of gold nanoparticles by small molecule thiols such as mercaptoethanol. (b) Representative cryoTEM micrograph of a dendrimicelle sample made from a G6-Au fraction of 0.3 after the addition of thiol. The micrograph shows that the dendrimicelles are still intact after thiol-induced etching of the micelle-embedded gold nanoparticles. (c) The number-averaged DLS size plot of dendrimicelles made from a fraction G6-Au of 0.3 after addition of thiol, indicates the micelle hydrodynamic diameter to be $\sim 50 \mathrm{~nm}$. This size plot suggests that the micelle diameter is not influenced by the addition of mercaptoethanol. (d) FRET efficiency plot showing the determined FRET efficiency of micelles made using a fluorophore-functionalized dendrimer fraction of 0.7 (i.e., $0.35 \mathrm{G} 6-\mathrm{F}$ and $0.35 \mathrm{G} 6-\mathrm{R}$ ) with the remaining fraction ( $f=0.3$ ) composed of various ratios of G6-E : G6-Au. The determined FRET efficiencies of dendrimicelles before mercaptoethanol addition are shown in black, while those of the same sample after incubation with mercaptoethanol are shown in orange. Clearly, the FRET efficiency of dendrimicelles containing gold nanoparticles recovers after the addition of thiol, indicating that the gold particles are destroyed by the action of mercaptoethanol. Consequently, the luminescence of the fluorophore-functionalized dendrimers is affected less, yielding an increase in FRET efficiency. Note: all samples were incubated with the same relative amount of mercaptoethanol (at least 6 molar equivalents to Au present). (e) Normalized emission spectra of the dendrimicelle sample made with a fraction G6-Au of 0.3 , before (black) and after (orange) the addition of mercaptoethanol. The increase in FRET-acceptor (G6-R) signal at $\sim 580 \mathrm{~nm}$ suggests an increase in FRET efficiency. (f) Photograph of the dendrimicelle sample made with a G6-Au fraction of 0.3, before (left), and after (right) incubation with mercaptoethanol. This photo shows that the perceived color of the micelles changes from green to yellow, and visualizes the FRET recovery from less FRET (left, with AuNPs present) to more FRET (right, AuNPs are (partially) destroyed). 
before (left) and after (right) thiol-mediated etching. By eye, the expected color difference is already visible, further corroborating spectral differences also observed in Fig. 4e.

\section{Conclusions}

Coacervate-core micelles constitute a class of relatively welldefined superstructures, and in particular dendrimeric subcomponents have great potential for final applications as well as systematic investigation of the nanoaggregates. In fact, in this work we have exploited the fluorescence properties and energy transfer between fluorescent molecules immobilized on different dendrimers, and their interaction with gold nanoparticles embedded in other dendrimers, revealing unambiguously and quantitatively the co-assembly of different types of subcomponents in one singular micellar core. More specifically, we developed four building blocks: non-functionalized, fluorescein-, and rhodamine-functionalized dendrimers, as well as dendrimer-encapsulated nanoparticles. With these building blocks we proved the formation of complex coacervate core micelles with a binary, ternary, and quaternary dendrimer core composition.

Co-assembly of both fluorophore-functionalized dendrimers results in FRET, the efficiency of which can be tuned not only by the ratio of the number of matching fluorescent dendrimers (i.e., the composition of the neighboring dendrimers) inside the micelle core, but also by increasing their spacing through co-assembly with non-fluorescent dendrimers forming micelles with a ternary core composition. Plasmonic coupling by gold nanopartcles decreased the FRET efficiencies, while thiol-induced etching of the gold nanoparticles yielded a recovery of the FRET efficiency, proving the quaternary core composition of the micelles. The micelle emission color and/ or FRET efficiency can potentially be used as sensor to identify the presence of, e.g., thiols or cyanide in solution. Considering this study used only 4 different G6 dendrimers, multiple follow-up studies can be envisioned. It is straightforward to design strategies to incorporate even more dendrimers variations (generations, types, functional groups) inside one micellar core. The concept could also be used in combination with complementary FRET pairs, different metal nanoparticles, and so forth. Well-defined systems are key to developing novel nanoscopic materials with emerging properties and the work presented here opens up a range of applications of these dendrimicelles in materials or medicinal areas. Controlling the micelle core composition also allows for the design of new supermolecule structures in which the micelles act as templates to allow chemical cross-linking of the core components, resulting in new classes of materials, such as tectodendrimers, ${ }^{56,57}$ or dendroids. ${ }^{58}$ For example, as welldefined multicompartment nanoreactors, towards encapsulation of drugs, for synthesizing stable nanocarriers, and as fluorescent tracer applications in biomedical applications, in multimodal diagnostic, therapeutic or theragnostic applications. $^{19,29,59}$

\section{Author contributions}

RK and JBtH contributed equally to this work. RK \& JBtH: conceptualization, research and writing; AB: writing; FWBvL: conceptualization and writing; AHV: conceptualization, research, supervision and writing.

\section{Conflicts of interest}

There are no conflicts to declare.

\section{Acknowledgements}

The Wageningen Electron Microscopy Centre, WEMC, is acknowledged for help with the cryoTEM measurements.

\section{References}

1 Z. B. Li, E. Kesselman, Y. Talmon, M. A. Hillmyer and T. P. Lodge, Science, 2004, 306, 98-101.

2 A. O. Moughton, M. A. Hillmyer and T. P. Lodge, Macromolecules, 2012, 45, 2-19.

3 N. Yoshinaga, T. Ishii, M. Naito, T. Endo, S. Uchida, H. Cabral, K. Osada and K. Kataoka, J. Am. Chem. Soc., 2017, 139, 18567-18575.

4 H. Cabral, K. Miyata, K. Osada and K. Kataoka, Chem. Rev., 2018, 118, 6844-6892.

5 C. V. Synatschke, T. Nomoto, H. Cabral, M. Fortsch, K. Toh, Y. Matsumoto, K. Miyazaki, A. Hanisch, F. H. Schacher, A. Kishimura, N. Nishiyama, A. H. E. Muller and K. Kataoka, ACS Nano, 2014, 8, 1161-1172.

6 A. H. Groschel, F. H. Schacher, H. Schmalz, O. V. Borisov, E. B. Zhulina, A. Walther and A. H. E. Muller, Nat. Commun., 2012, 3, 710.

7 J. Z. Du and R. K. O'Reilly, Chem. Soc. Rev., 2011, 40, 24022416.

8 S. Kubowicz, J. F. Baussard, J. F. Lutz, A. F. Thunemann, H. von Berlepsch and A. Laschewsky, Angew. Chem., Int. Ed., 2005, 44, 5262-5265.

9 I. K. Voets, A. de Keizer and M. A. Cohen Stuart, $A d v$. Colloid Interface Sci., 2009, 147-148, 300-318.

10 J. R. Magana, C. C. M. Sproncken and I. K. Voets, Polymers, 2020, 12, 1953.

11 A. K. Harada, Macromolecules, 1998, 31, 288-294.

12 J. Y. Wang, A. H. Velders, E. Gianolio, S. Aime, F. J. Vergeldt, H. Van As, Y. Yan, M. Drechsler, A. de Keizer, M. A. C. Stuart and J. van der Gucht, Chem. Commun., 2013, 49, 3736-3738.

13 J. Y. Wang, A. Groeneveld, M. Oikonomou, A. Prusova, H. Van As, J. W. M. van Lent and A. H. Velders, Soft Matter, 2016, 12, 99-105.

14 A. Hernandez-Garcia, A. H. Velders, M. A. C. Stuart, R. de Vries, J. W. M. van Lent and J. Wang, Chem. - Eur. J., 2017, 23, 239-243. 
15 K. Kataoka, A. Harada and Y. Nagasaki, Adv. Drug Delivery Rev., 2001, 47, 113-131.

16 G. Gaucher, M.-H. Dufresne, V. P. Sant, N. Kang, D. Maysinger and J.-C. Leroux, J. Controlled Release, 2005, 109, 169-188.

17 J. Ding, L. Chen, C. Xiao, L. Chen, X. Zhuang and X. Chen, Chem. Commun., 2014, 50, 11274-11290.

18 C. Facciotti, V. Saggiomo, A. Bunschoten, R. Fokkink, J. B. ten Hove, J. Y. Wang and A. H. Velders, Soft Matter, 2018, 14, 9542-9549.

19 S. Mignani, X. Y. Shi, M. Zablocka and J. P. Majoral, Biomacromolecules, 2021, 22, 262-274.

20 D. A. Tomalia, Prog. Polym. Sci., 2005, 30, 294-324.

21 M. Florendo, A. Figacz, B. Srinageshwar, A. Sharma, D. Swanson, G. L. Dunbar and J. Rossignol, Molecules, 2018, 23, 2238.

22 L. Z. Lin, Y. Fan, F. Gao, L. F. Jin, D. Li, W. J. Sun, F. Li, P. Qin, Q. S. Shi, X. Y. Shi and L. F. Du, Theranostics, 2018, 8, 1923-1939.

23 R. M. Anderson, D. F. Yancey, L. Zhang, S. T. Chill, G. Henkelman and R. M. Crooks, Acc. Chem. Res., 2015, 48, 1351-1357.

24 F. Gröhn, B. J. Bauer, Y. A. Akpalu, C. L. Jackson and E. J. Amis, Macromolecules, 2000, 33, 6042-6050.

25 R. W. J. Scott, O. M. Wilson and R. M. Crooks, J. Phys. Chem. B, 2005, 109, 692-704.

26 V. M. Gomez, J. Guerra, S. V. Myers, R. M. Crooks and A. H. Velders, J. Am. Chem. Soc., 2009, 131, 14634-14635.

27 V. M. Gomez, J. Guerra, A. H. Velders and R. M. Crooks, J. Am. Chem. Soc., 2009, 131, 341-350.

28 X. Y. Shi, S. H. Wang, S. Meshinchi, M. E. Van Antwerp, X. D. Bi, I. H. Lee and J. R. Baker, Small, 2007, 3, 1245-1252.

29 A. M. Caminade, A. Hameau, C. O. Turrin, R. Laurent and J. P. Majoral, Coord. Chem. Rev., 2021, 430, 213739.

30 A. Kakkar, G. Traverso, O. C. Farokhzad, R. Weissleder and R. Langer, Nat. Rev. Chem., 2017, 1, 0063.

31 P. Wen, X. Y. Wang, S. Moreno, S. Boye, D. Voigt, B. Voit, X. Huang and D. Appelhans, Small, 2021, 17, 2005749.

32 A. H. Groschel and A. H. E. Muller, Nanoscale, 2015, 7, 11841-11876.

33 J. Wang, I. K. Voets, R. Fokkink, J. van der Gucht and A. H. Velders, Soft Matter, 2014, 10, 7337-7345.

34 J. B. ten Hove, J. Wang, M. N. van Oosterom, F. W. B. van Leeuwen and A. H. Velders, ACS Nano, 2017, 11, 1122511231.

35 J. B. ten Hove, M. N. van Oosterom, F. W. B. van Leeuwen and A. H. Velders, Sci. Rep., 2018, 8, 13820.

36 J. B. ten Hove, F. W. B. van Leeuwen and A. H. Velders, Nat. Commun., 2018, 9, 5207.

37 J. B. ten Hove, J. Wang, F. W. B. van Leeuwen and A. H. Velders, Nanoscale, 2017, 9, 18619-18623.
38 K. H. Ku, M. P. Kim, K. Paek, J. M. Shin, S. Chung, S. G. Jang, W. S. Chae, G. R. Yi and B. J. Kim, Small, 2013, 9, 2667-2672.

39 D. Dorokhin, S. H. Hsu, N. Tomczak, C. Blum, V. Subramaniam, J. Huskens, D. N. Reinhoudt, A. H. Velders and G. J. Vancso, Small, 2010, 6, 2870-2876.

40 H. Chen, Z. Y. Wang, S. F. Zong, P. Chen, D. Zhu, L. Wu and Y. P. Cui, Nanoscale, 2015, 7, 15477-15486.

41 A. Ruggi, C. Beekman, D. Wasserberg, V. Subramaniam, D. N. Reinhoudt, F. W. B. van Leeuwen and A. H. Velders, Chem. - Eur. J., 2011, 17, 464-467.

42 L. Albertazzi, B. Storti, L. Marchetti and F. Beltram, J. Am. Chem. Soc., 2010, 132, 18158-18167.

43 A. W. Bosman, H. M. Janssen and E. W. Meijer, Chem. Rev., 1999, 99, 1665-1688.

44 S. M. Grayson and J. M. J. Frechet, Chem. Rev., 2001, 101, 3819-3867.

45 D. Astruc, E. Boisselier and C. Ornelas, Chem. Rev., 2010, 110, 1857-1959.

46 M. W. Shen and X. Y. Shi, Nanoscale, 2010, 2, 1596-1610.

47 C. Wängler, G. Moldenhauer, R. Saffrich, E. M. Knapp, B. Beijer, M. Schnölzer, B. Wängler, M. Eisenhut, U. Haberkorn and W. Mier, Chem. - Eur. J., 2008, 14, 81168130 .

48 W. Schrimpf, J. C. Jiang, Z. Ji, P. Hirschle, D. C. Lamb, O. M. Yaghi and S. Wuttke, Nat. Commun., 2018, 9, 1647.

49 D. G. Mullen, A. Desai, M. A. van Dongen, M. Barash, J. R. Baker and M. M. Banaszak Holl, Macromolecules, 2012, 45, 5316-5320.

50 M. A. van Dongen, A. Desai, B. G. Orr, J. R. Baker and M. M. Banaszak Holl, Polymer, 2013, 54, 4126-4133.

51 G. W. Gordon, G. Berry, X. H. Liang, B. Levine and B. Herman, Biophys. J., 1998, 74, 2702-2713.

52 S. K. Ghosh and T. Pal, Chem. Rev., 2007, 107, 4797-4862.

53 F. Tam, G. P. Goodrich, B. R. Johnson and N. J. Halas, Nano Lett., 2007, 7, 496-501.

54 C. Facciotti, V. Saggiomo, A. Bunschoten, J. B. ten Hove, M. T. Rood, F. W. van Leeuwen and A. H. Velders, ChemSystemsChem, 2020, 2, e1900032.

55 C.-Y. Ke, T.-H. Chen, L.-C. Lu and W.-L. Tseng, RSC Adv., 2014, 4, 26050-26056.

56 S. Uppuluri, D. R. Swanson, L. T. Piehler, J. Li, G. L. Hagnauer and D. A. Tomalia, Adv. Mater., 2000, 12, 796-800.

57 M. Studzian, P. Dzialak, L. Pulaski, D. M. Hedstrand, D. A. Tomalia and B. Klajnert-Maculewicz, Molecules, 2020, 25, 4406.

58 R. Kaup, J. B. Ten Hove and A. H. Velders, ACS Nano, 2021, 15, 1666-1674.

59 C. Song, M. W. Shen, J. Rodrigues, S. Mignani, J. P. Majoral and X. Y. Shi, Coord. Chem. Rev., 2020, 421, 213463. 